\title{
Por arte de barrio. Los jóvenes voluntarios de la ' $Y$ ' y sus acciones colectivas en la Comuna 13 de Medellín ${ }^{1}$ By virtue of the neighbourhood. Voluntary youth at the ' $Y$ ' and their collective actions in Medellin's Commune 13
}

\author{
Daniela Franco Pedraza \\ Universidad del Rosario \\ fpdaniela9@gmail.com
}

\section{Historia editorial \\ Recibido: 16/02/2021 \\ Aceptado: 14/05/2021 \\ Publicado: 15/05/2021 \\ Palabras clave \\ Comuna 13; Territorio; \\ Acciones colectivas; \\ Jóvenes; Voluntariado; ACJ \\ Medellín}

\section{Resumen}

Este artículo busca mostrar la importancia que tiene la juventud en el momento de transformar la comunidad e incidir en ella. Lo anterior, tomando el caso particular de las acciones colectivas que se llevan a cabo por los jóvenes que pertenecen al voluntariado de la ACJ (Asociación Cristiana de Jóvenes) Medellín, ubicada en un lugar anteriormente marcado por la violencia como lo es la Comuna 13. En el voluntariado se busca que los jóvenes de la Comuna 13 fortalezcan sus capacidades como individuos estimulando estas a través de metodologías experienciales y metodologías de educación popular de enseñanza, logrando así convertir a estos jóvenes en líderes que replican diferentes acciones en cada uno de los barrios de su Comuna.

\section{Abstract}

This article aims to show the importance of youth when transforming the community and influencing it. This is done by studying the particular case of the collective actions carried out by young people belonging to the volunteer work of the YMCA (Young Christian Association) Medellin, located in a place troubled by violence such as Commune 13. The Voluntary service which is implemented intends the youth in Commune 13 to strengthen their capacities as individuals, stimulating these through experiential methodologies and popular education methodologies of teaching. In this way, these young people are turned into leaders who replicate different actions in each of the neighborhoods in their own Communes.

Franco Pedraza, D. (2021). Por arte de barrio. Los jóvenes voluntarios de la ' $Y$ ' y sus acciones colectivas en la Comuna 13 de Medellín, Clivatge, 9, e- 33756 https://www.doi.org/CLIVATGE2021.9.5

1 Este artículo de investigación es derivado del trabajo de grado de Sociología en la Universidad del Rosario, de la autora. Trabajo dirigido por: Carlos Andrés Charry Joya. PhD. en Sociología. 


\section{Introducción}

Medellín ha sido una ciudad que se ha visto afectada por distintos procesos de violencia, por lo que anteriormente era reconocida como un "espacio de creación, asentamiento y recepción de bandas, combos y pandillas" (Moreno, 2003, 220). Entre los lugares más afectados de la ciudad se encuentra la Comuna 13, en donde, para los años 90, se tuvo la presencia simultánea de grupos armados como las Fuerzas Armadas Revolucionarias de Colombia (FARC), el Ejército de Liberación Nacional (ELN), los Comandos Armados del Pueblo (CAP) y grupos paramilitares, tales como las Autodefensas Unidas de Colombia (AUC). Los enfrentamientos entre estos grupos armados y la búsqueda del dominio territorial y político obstaculizaron expresiones libres del ejercicio ciudadano y las manifestaciones propias de la cotidianidad (Moreno, 2003).

La Comuna 13, de acuerdo con el Centro Nacional de Memoria Histórica, es un lugar que, desde mediados de la década de 1990, se ha caracterizado por recibir un número considerable de desplazados, que llegaron a esta región urbana tratando de huir de diferentes formas de violencia en el país. En consecuencia, el proceso de urbanización de la Comuna se dio a partir de la ocupación informal del espacio y de la invasión (CNMH, 2011). Al estar ubicada en el centro-occidente de Medellín y tener cercanía con la carretera al mar -vía que comunica Medellín con el puerto de Urabá-, la Comuna se convirtió en un espacio clave dentro de los modelos de desarrollo regional que se tenían a comienzo de siglo. Sin embargo, esa ubicación estratégica también fue aprovechada por los grupos armados que se encontraban en la zona, pues la Comuna fue tomada como "corredor estratégico para el transporte de droga, el ingreso de armas, el traslado de integrantes de frentes rurales hacia la ciudad, el refugio de personas buscadas por la justicia y para mantener secuestrados" (CNMH, 2011, 56).

A partir de 2002, comenzaron a evidenciarse intentos por la recuperación de la Comuna 13, luego de la toma del control de esta, la Comuna empezó a ser el foco de intervenciones planeadas desde 
la Alcaldía de Medellín que tenían por objetivo la mejora de las condiciones de vida de sus habitantes. Entre esas intervenciones se encuentran: el metro cable, que entró en funcionamiento en el 2004; las escaleras eléctricas que operan desde el 2011; el Programa Integral de Mejoramiento de Barrios Informales (PMIB) que buscaba un reordenamiento urbano de la vivienda, mitigación del riesgo y mejoramiento de la habitabilidad (Alcaldía de Medellín, 2015); y las intervenciones artísticas en el espacio público, en donde se destaca un trabajo colectivo de acciones políticas para recuperar no solo el espacio físico, por medio del embellecimiento o modernización del sector, sino también la reconstrucción del tejido social de mano de la memoria y la colectividad.

A partir de las intervenciones mencionadas se han ido transformado las dinámicas de la Comuna 13, se empieza a facilitar el acceso a la zona para los habitantes y externos que ahora tienen el interés de visitar este espacio. Desde entonces, se han creado múltiples asociaciones que buscan mostrar una cara artística, cultural, esperanzadora y resiliente de la Comuna 13, las iniciativas que han surgido, en su mayoría, han sido por parte de jóvenes que empiezan a llevar a cabo acciones colectivas. Demostrando así, que son agentes sociales dueños de sus proyectos de vida y que motivando nuevas formas de acción logran contrarrestar las situaciones de violencia y exclusión social que han tenido que vivir (Álzate, 2012).

Este artículo se divide en seis secciones: introducción, planteamiento del problema, metodología, resultados, con tres subsecciones, conclusiones y finalmente bibliografía. Lo que busca este artículo es poder visibilizar las acciones que se llevan a cabo por los jóvenes de la ' $\mathrm{Y}$ ' y la forma en la que un lugar marcado por la violencia puede empezar a ser resiliente.

\section{Planteamiento del problema}

Entre las asociaciones que más se reconocen en la Comuna 13, se encuentra la ACJ-YMCA (Asociación Cristiana de Jóvenes - Young Men Christian Association). Este movimiento internacional surge en 1844 bajo el propósito de mejorar la condición espiritual de los y las 
jóvenes que trabajaban en las grandes fábricas de la revolución industrial. Actualmente, se encuentra en cerca de 11.000 comunidades del mundo, cuenta con presencia en 119 países y tiene cerca de 700.000 voluntarios. En 1855, con la declaración fundacional del movimiento YMCA se establece que el énfasis principal va a ser la formación de la población joven y a partir de esto se va a buscar la construcción de una comunidad humana de justicia, paz y reconciliación. Asimismo, se establece que cada YMCA deberá enfocarse en los problemas particulares de cada uno de sus contextos. (Consejo Mundial de ACJs, 1998.)

A Colombia la YMCA llega en 1963 y en la ciudad de Medellín es fundada en 1974 ubicándose en un lugar aledaño a la ciudad. Para los años 90, la ACJ-YMCA Medellín se traslada a la Comuna 13 y cumple un papel importante allí, pues durante los años de auge de la violencia y el narcotráfico la formación por parte de la ACJ-YMCA Medellín se enfoca en la creación de proyectos de vida, el reconocimiento del otro como igual, la dignificación del ser humano, entre otros objetivos, intentando alejar a la población joven de dichas problemáticas. Asimismo, la ACJ-YMCA Medellín empieza a mediar entre la comunidad y la administración municipal para la ejecución de programas de formación. (ACJ-YMCA, (s.f.))

Los principales propósitos de la ACJ-YMCA Medellín se centran en una apuesta por la paz y la reconciliación, el empoderamiento juvenil y comunitario, el fortalecimiento del voluntariado, la viabilidad y desarrollo institucional y la gestión ambiental (ACJYMCA, 2017, 10). El papel prioritario de la juventud dentro de la ACJ-YMCA Medellín tiene que ver con que los y las jóvenes han adquirido un papel cada vez más significativo dentro de la sociedad, pues son ellos quienes constantemente están cuestionando la realidad y quienes cada vez más están haciendo esfuerzos por pasar de ser sujetos pasivos a ser sujetos activos dentro de la toma de decisiones para la construcción de mejoras en sus propias vidas y en la de sus comunidades (Consejo Mundial de Kampala, 1973). 
Este artículo tiene el propósito de mostrar cómo a partir del voluntariado de la ACJ-YMCA Medellín se contribuye a la transformación de la Comuna 13. Explorando el proceso de formación que implica el voluntariado y mencionando algunas de las actividades más representativas que se han realizado en la asociación en el último año. La investigación se basa en un acercamiento de campo a la casa de la ' $\mathrm{Y}$ ' Medellín, en donde se realizó observación participante y no participante, entrevistas a varios de los voluntarios y a las directivas de la ' $Y$ '. A su vez, se desarrollaron diferentes talleres de aprendizaje colectivo como cartografías sociales y grupos focales, que permitieron arrojar conclusiones a la investigación. Los resultados se obtuvieron a través de un abordaje de corte cualitativo, en el que se hizo un trabajo de campo que permitió la recolección de información y de diferentes conclusiones, esto, para que la investigación no fuera estática y se pudiera enriquecer desde diferentes aspectos los resultados de la investigación.

\section{Metodología}

En cuanto a las etapas de la investigación, en un primer momento se realizó una revisión bibliográfica para tener claridad sobre el contexto del lugar a investigar, posteriormente se realizó una investigación en campo sobre las asociaciones de jóvenes más representativas de la Comuna 13, ejercicio que permitió elegir la ACJ como estudio de caso. En un tercer momento, se empezó el trabajo de campo, de la mano de la coordinadora del voluntariado de la ACJ Medellín, Manuela Henao, en donde se coordinaron y se planearon talleres en conjunto para realizar con los y las jóvenes voluntarias para entender cuáles son sus objetivos, cómo nacen las iniciativas, cómo se conforman, cómo dividen su trabajo, cuáles son sus intereses a corto, mediano y largo plazo, cómo funciona la ' $\mathrm{Y}$ ', el voluntariado, qué acciones han realizado, etc. En medio de este proceso se pudo acompañar a los jóvenes del voluntariado en algunas de las actividades que realizaron en el territorio. En un cuarto momento, se organizó y clasificó la información recolectada. 
Y, por último, luego de la clasificación de la información se empezó a construir el artículo de investigación.

El interés que tengo como investigadora, se basa en el impacto que tuvo para mí conocer las diferentes formas en la que los habitantes de la Comuna 13 -en su mayoría jóvenes- han buscado diferentes salidas por medio del arte, la música, el baile e incluso la educación y formación, a todos los conflictos en los que cotidianamente se ven envueltos. Esto sin importar las condiciones en las que ellos se encuentren. Si bien es importante reconocer que las realidades de muchos jóvenes se han transformado, no se puede dejar de lado los conflictos que aún están latentes en los diferentes barrios de la Comuna, que hacen que llevar a cabo estas acciones sea algo mucho más complejo.

\section{Resultados}

\subsection{Reconociendo el territorio: la Comuna 13}

El barrio San Javier o la Comuna 13 de Medellín está conformada por 20 barrios: El Pesebre, Blanquizal, Santa Rosa de Lima, Los Alcázares, Metropolitano, La Pradera, Juan XXIII, Antonio Nariño, San Javier n.․ㅜ 1, San Javier n.ํㅜ 2, Veinte de Julio, El Salado, Nuevos Conquistadores, Las Independencias (I y II), El Corazón, Belencito, Betania, La Divisa, Eduardo Santos y El Socorro. 
Figura 1: Mapa Comuna 13 de Medellín

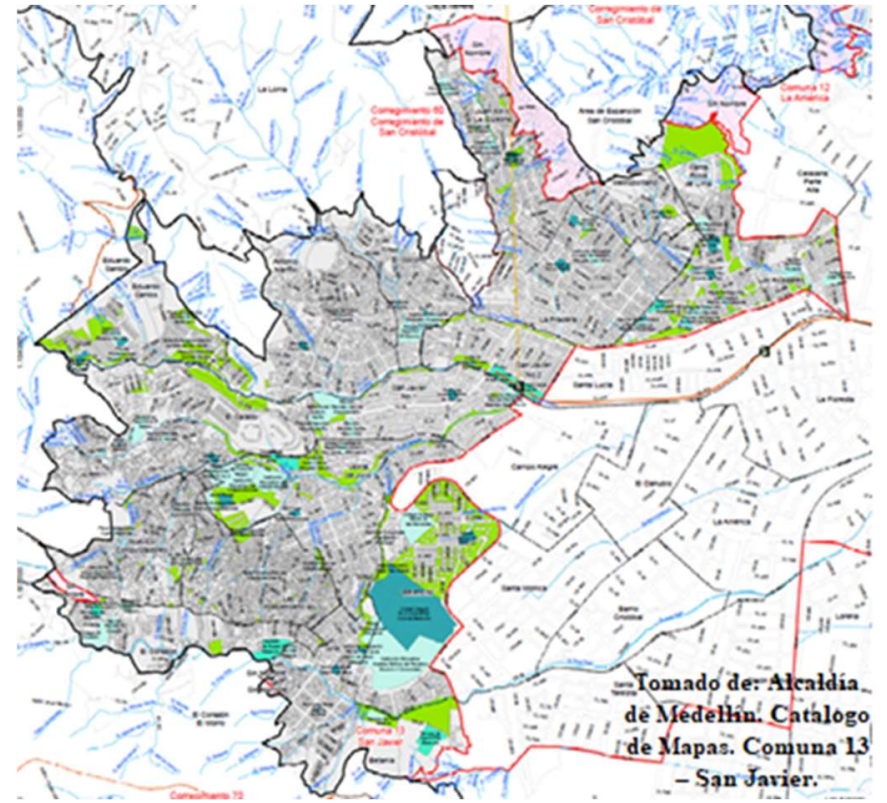

Fuente: Alcaldía de Medellín. Catálogo de Mapas. Comuna 13 - San Javier

Desde los años de más auge de conflicto, la Comuna 13 ha sido el foco de diferentes intervenciones y podría afirmarse también que ha sido uno de los lugares del país en donde más se ha concentrado el conflicto armado y las diferentes modalidades de violencia se han asentado. El narcotráfico, los repertorios de violencia contra los civiles usados por la guerrilla -el secuestro- y el desarrollo cada vez más amplio y poderoso de modalidades de provisión privada de seguridad, son según Gutiérrez (2015) los factores que permitieron que las guerrillas sobrevivieran durante tanto tiempo en el país.

A partir de 2002 y durante la alcaldía de Luis Pérez Gutiérrez, comenzaron a evidenciarse intentos por la recuperación de la Comuna 13, sin embargo, no se lograron avances significativos. Posteriormente, el Estado empezó a intervenir y entre las acciones más destacadas sobre la Comuna se encuentra la Operación Orión que tuvo lugar en octubre del mismo año, bajo la presidencia de Álvaro Uribe Vélez. Esta operación tenía el objetivo de recuperar la Comuna 13 del control de los grupos armados, sin embargo, el operativo militar fue muy cuestionado y es conocido como "el 
combate urbano más cruento, intenso y prolongado que se haya registrado en la historia del país" (Verdad abierta, 2018). Según datos del CINEP, como resultado de dicha operación se produjo la muerte de aproximadamente 88 civiles, 80 heridos, 370 detenciones arbitrarias y 95 desaparecidos de manera forzada (Cinep \& Justicia y Paz, 2003).

Lindón (2009) afirma que existen lugares en los cuales algunos sujetos ejercen o han ejercido la violencia en otros tiempos -caso de la Comuna 13-, estos lugares, se tiñen con esa violencia ejercida y constituyen una memoria del lugar, la violencia configura el lugar a través de la memoria espacial. En efecto, la Comuna 13 presenta una memoria de violencia, pero también, a partir de la unión de diferentes acciones colectivas llevadas a cabo por asociaciones que han surgido con el vínculo de los habitantes de la Comuna se ha generado la transformación de dicho espacio, pues, "el espacio urbano representa para el hombre la tentativa más coherente y, en general, la más satisfactoria de recrear el mundo en que vive de acuerdo a su propio deseo (...) al crear la ciudad el hombre se recrea a sí mismo" (Park, 1999, 115).

Es por esto, que incluso en las épocas de mayor enfrentamiento, los habitantes de la Comuna 13 han sido reconocidos y se han caracterizado por la búsqueda de opciones diferentes que puedan contrarrestar de alguna forma las pugnas que se dan en y por el territorio, así como han luchado porque la Comuna 13 no sea reconocida por dichas memorias de violencia. En la Comuna 13 han surgido diversas asociaciones cuyo objetivo principal ha sido generar, como afirma Park (1999), un sentimiento local, pues de la residencia parte la base de la participación en asuntos públicos y esta vecindad -la Comuna 13- se puede entender como una unidad social y como un espíritu colectivo. Asimismo, es importante "comprender la construcción social de la ciudad, de lo urbano y sus lugares, a través de los sujetos que la habitan corporal y emocionalmente" (Lindón, 2009, 6). Para la sociología, el valor del espacio se define en términos de su carácter estructurante, pues organiza nuestras percepciones y las que tenemos de los fenómenos 
sociales, a la vez que es portador de acontecimientos sociales y de expresiones sobre la sociedad (Leal, 1997).

Actualmente, la Comuna 13 ha logrado no solo en el país, sino también en el exterior, ser reconocida como símbolo de transformación a través del arte y la cultura. Periódicos nacionales como El Tiempo, El Espectador y Semana resaltan entre sus titulares aspectos positivos de la Comuna 13: "Graffitour, un recorrido por la transformación de la comuna 13” (El Tiempo, 2016), "La Comuna 13 de Medellín exhibe su renacer ante autoridades locales del mundo" (El Espectador, 2019), "Comuna 13: "En este lugar florecen el turismo, la autonomía y la integración social" (Semana Video, 2020). A pesar de que la Comuna ha mejorado en muchos aspectos aún existen problemas que según los habitantes son "invisibilizados". Los y las jóvenes de la ACJ Medellín, que viven en alguno de los barrios de la Comuna, lograron identificar en un taller de cartografía social que entre los problemas principales que se viven en la actualidad se encuentra el consumo de sustancias psicoactivas, pues mencionan que existe un fácil acceso a ellas en la mayoría de los barrios en dónde viven. Los barrios en los que se elaboró este ejercicio fueron: El Socorro, 20 de Julio, Nariño, San Javier, y El Salado. 


\section{Figura 2: Cartografía barrio El Socorro.}

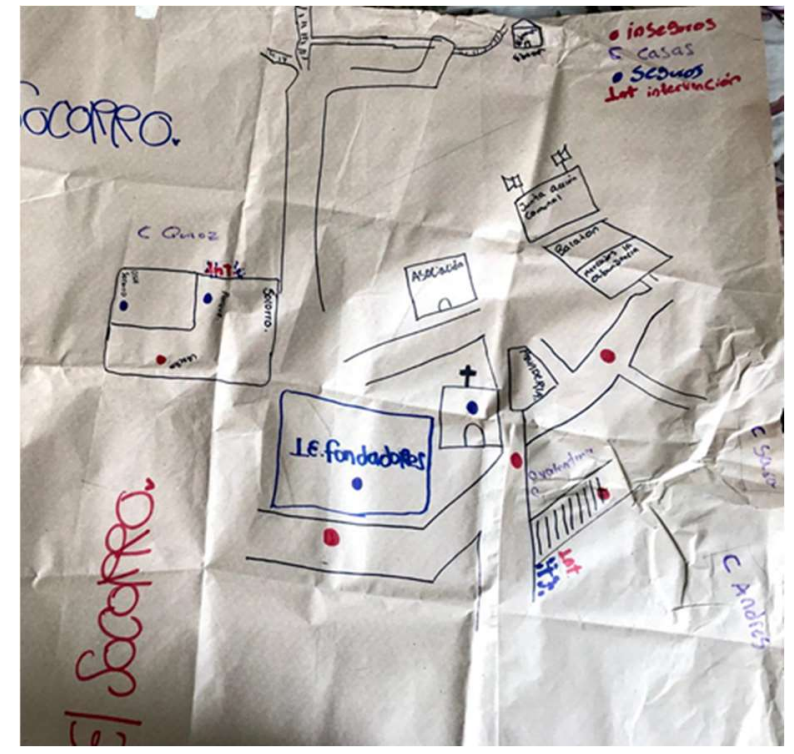

Fuente: Taller de Cartografía realizado con los jóvenes de la ACJ el 29 de Julio 2019.

El barrio El Socorro, es un lugar con mucho tránsito y constantemente se generan deslizamientos de tierras por las lluvias. A su vez, en una parte del barrio se acumulan basuras y la zona se reconoce como insegura. Existen también puntos que fueron identificados como "puntos de venta de drogas" y este negocio es reconocido como el principal, uno de estos puntos de venta se encuentra en las afueras del colegio del barrio. Si bien se cuenta con la presencia de algunos policías en la zona, los y las jóvenes manifiestan que no se le presta la suficiente atención a este negocio ilegal. Por otro lado, entre las zonas seguras, se reconoce el interior de la sede secundaria del colegio y la Junta de Acción Comunal del barrio que es un punto de encuentro de todos los y las jóvenes que allí habitan para hacer diversas actividades. La ACJ ha tenido presencia en el barrio con el voluntariado.

A partir de un mapeo sobre las violencias que vive la Comuna 13 que compartió en la socialización, una de las voluntarias de la ACJ, Valeria, en el barrio 20 de Julio, se puede identificar que se 
reconocen las escaleras eléctricas como un lugar que necesita intervención, pues si bien ayuda a mejorar el acceso hacia las zonas más retiradas de la Comuna, también es un espacio que se está usando para el consumo de sustancias y que ha revivido el problema del turismo sexual. Al ser reconocido como un lugar que hay que recorrer cuando se va a Medellín, la Comuna 13 no solo es visitada por personas que tienen la intención de conocer la historia detrás de la transformación, sino también, la Comuna es visitada por turistas que muchas veces van en búsqueda de mujeres y drogas.

\section{Figura 3: Cartografía barrio 20 de Julio}

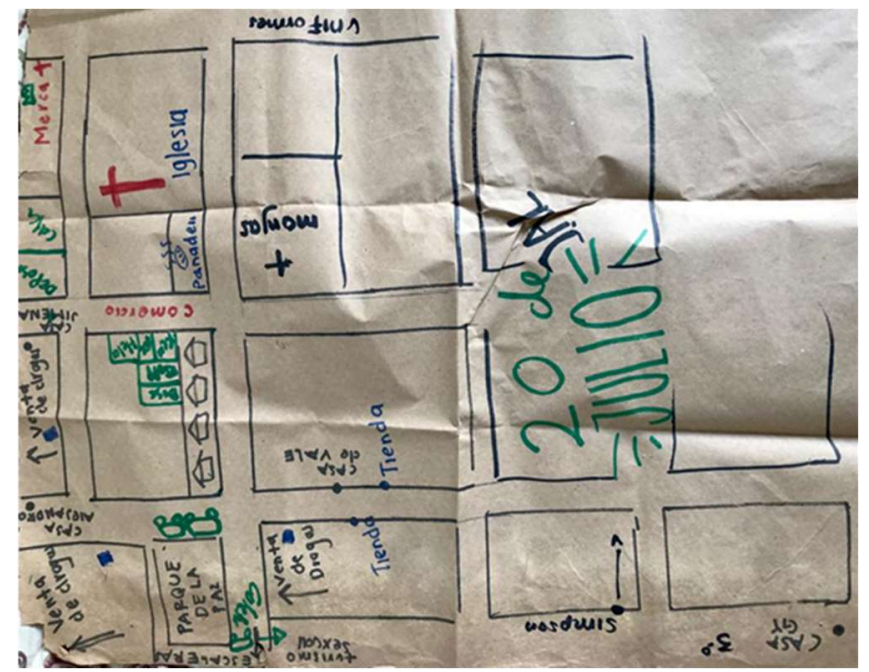

Fuente: Taller de Cartografía realizado con los jóvenes de la ACJ el 29 de Julio 2019

Asimismo, con el negocio del turismo se ha buscado regular el tema de quién puede o no realizar estos tours por el barrio. Los habitantes de la Comuna piden que sean únicamente ellos quiénes puedan ofrecer estos servicios a los turistas, de modo que puedan contar con sus palabras y bajo sus vivencias particulares lo que ha sido pasar de estar en medio del conflicto a ser parte de la transformación. Por esta razón, en las escaleras eléctricas se creó una mesa de turismo conformada por las organizaciones de la Comuna 13 y otras tres organizaciones de turismo de Medellín. Dicha mesa de turismo busca retribuir por medio de todas las ganancias que generan los 
tours algo al territorio, esperando hacer alguna intervención o usar las ganancias para concretar algo que beneficie a toda la comunidad.

Por su parte, en el barrio Nariño también se identifica el problema de acumulación de basuras, los lugares que se reconocen como inseguros se centran en los parques del barrio, detrás de la Iglesia en un lugar que llaman "el hueco" y los lugares seguros son nuevamente la Iglesia del Divino Niño y la Junta de Acción Comunal. En el barrio San Javier, barrio cercano a la estación del metro, las zonas reconocidas como seguras son: la estación del metro, la biblioteca y el centro del barrio, se reconocieron estos lugares como seguros por la presencia de muchas personas durante casi todo el día. Como inseguros, se reconoce la cancha del metro pues según los y las jóvenes hay presencia de bandas delincuenciales. Por último, el barrio El Salado es un barrio que tiene muchas lomas y esto hace que existan muchos lugares en donde se esconden las bandas, al lado de la casa de varios de los voluntarios que viven en este barrio venden drogas y el parque UVA es uno de los lugares en donde más se consume y existe mucha contaminación auditiva.

\section{Figura 4: Cartografía barrio Nariño}

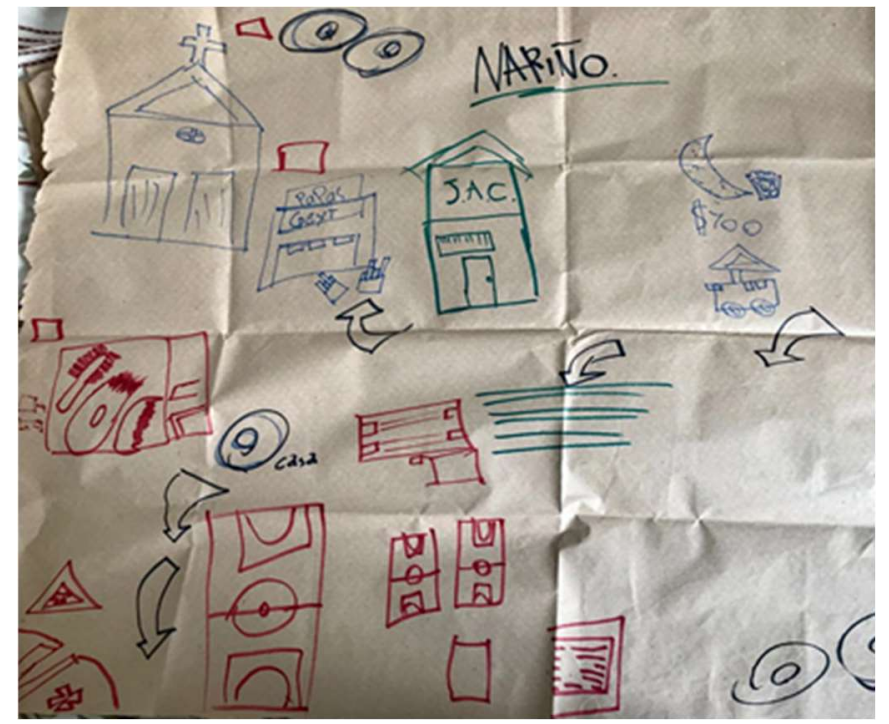

Fuente: Taller de Cartografía realizado con los jóvenes de la ACJ el 29 de Julio 2019 


\section{Figura 5: Cartografía barrio San Javier.}

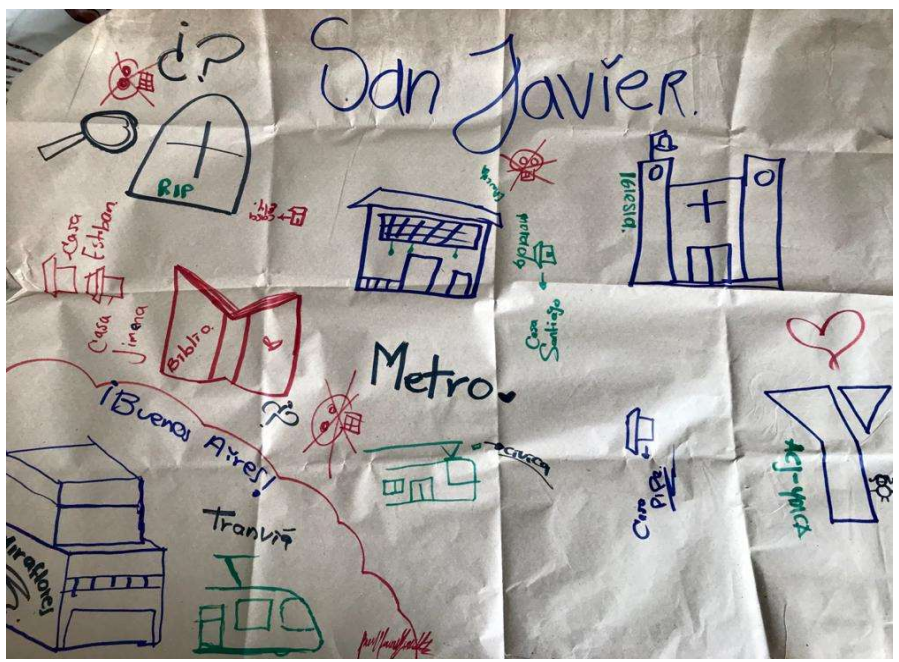

Fuente: Taller de Cartografía realizado con los jóvenes de la ACJ el 29 de Julio 2019

\section{Figura 6: Cartografía barrio Salado}

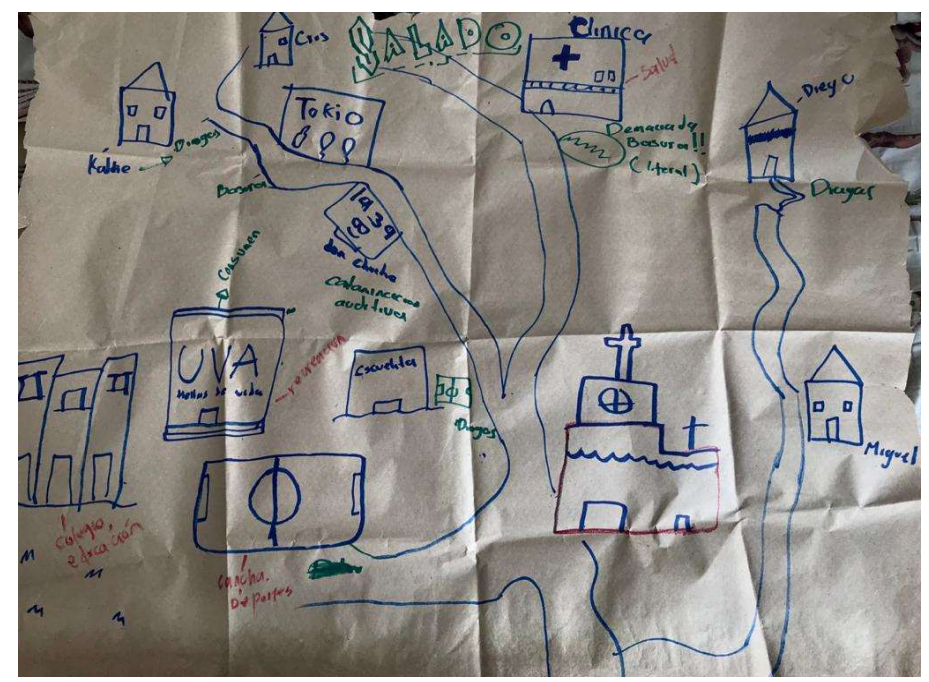

Fuente: Taller de Cartografía realizado con los jóvenes de la ACJ el 29 de Julio 2019

Después de reconocer puntos seguros, inseguros y que necesitan intervención en cada uno de los barrios mencionados, se concluyó que todos los y las jóvenes tienen problemas en común, además, este ejercicio aportó para que se reduzcan los estigmas que ellos mismos 
tienen sobre otros barrios de la Comuna, esto a través de la construcción conjunta que se logró hacer destacando en dónde se encuentra el potencial en cada barrio. Es importante mencionar que entre los lugares que se identifican como seguros para todos los y las jóvenes se incluye la casa de la ACJ. Asimismo, reconocen que las acciones que realizan como voluntarios y voluntarias son las que más han aportado a la reducción de problemáticas en sus mismos barrios y comunidades.

Como menciona Urán (2002) el reconocer un espacio público deteriorado y percibirlo como inseguro, tanto física como socialmente, reduce las posibilidades de la gente joven para estar fuera de casa. Como se resaltó anteriormente existen aún problemas relacionados con la venta de sustancias psicoactivas, el turismo sexual, el cobro de vacunas y la violencia hacia las mujeres dentro de la Comuna. Sin embargo, entre lo que era la Comuna 13 antes y lo que es ahora, según los y las jóvenes voluntarias, sí pueden identificarse cambios y muchos logran resaltar aspectos positivos enfocándose en ello para seguir generando transformaciones no solo en el espacio público sino también en los individuos desde su papel como voluntarios:

Yo digo que rescato de la Comuna 13, que es una Comuna colorida, diversa, una Comuna muy diferente a los imaginarios de las otras personas, rescato que es una Comuna de personas muy líderes, de personas que quieren salir adelante, que buscan una resistencia frente a las violencias que les sucedan y que esas resistencias hacen personas resilientes. Entonces digo que mi Comuna 13 es arte, cultura, educación. (Entrevista Personal, Valeria Gutiérrez, 15 años. Voluntaria ACJ.)

En la actualidad, los y las jóvenes de la Comuna 13 que pertenecen a la ACJ están adquiriendo un papel cada vez más protagónico en cada uno de sus barrios. Cuestionando constantemente sus realidades, enfrentando y reconociendo las problemáticas que se presentan en los barrios en donde viven para así poder planear acciones colectivas que permitan que su Comuna se convierta en un lugar 
ejemplo de resiliencia, buscando dejar atrás el pasado violento que ha marcado el territorio. Generando así, que empiecen a verse no solo como gestores de paz sino también como agentes de cambio, transformación social, política y cultural. Asimismo, es importante reconocer que "el crecimiento y desarrollo de la gente joven depende fuertemente de ambientes que le provean estimulación, le permitan autonomía, ofrezcan posibilidades para la exploración y promuevan el aprendizaje independiente" (Urán, 2002, pág. 155). De esta forma, la ACJ y los objetivos que se proponen se convierten en clave para la construcción de la identidad social de los jóvenes que pertenecen al voluntariado.

Los voluntarios que hicieron parte de la construcción de la cartografía social por barrios fueron: barrio El Socorro: Andrés Estiven Rueda Oquendo, 16 años. Elber Muñoz Blandón, 21 años, Joan Felipe Correa Rua, 16 años. Sara Parra Moncada, 17 años, Sebastián Andrés Quiroz Gomez, 19 años. Yeimy Carolina David Londoño, 19 años. Eduan Alonso Pérez López, 18 años. Sara Borja Ruiz, 15 años. Kellys Polo, 19 años. Isabel Parra López, 21 años. Barrio El Salado: Cristian Camilo Durango, 19 años. Danna Cristina Suarez Tejada, 23 años. Jimena Patiño Sepúlveda, 17 años. Katherine Mesa Bedoya, 17 años. Luis Miguel Caro Álvarez, 19 años. Diego Alejandro Acevedo García, 18 años. Barrio Nariño: Cristian Gutiérrez López, 16 años. Cristian Camilo Arcila Escobar, 17 años. Amir Flores Rojas, 18 años. Rodolfo Aicardo Orozco, 25 años. Sergio Agames Correa, 17 años. Barrio 20 de Julio: Valeria Gutiérrez, 16 años. Gypsyn Karolay Quiroz Quina, 16 años. Y barrio San Javier: Juan Esteban Higuita Montoya, 19 años. María Camila Higuita, 21 años. Eliana Marcela Carrillo Yarce, 15 años. Santiago Molina Ruiz, 17 años.

\subsection{La 'Y' Medellín y el voluntariado}

La ACJ Medellín -o la 'Y' en inglés, como la llaman los voluntariostiene como objetivo principal enfocarse en el desarrollo de valores y 
principios para los y las jóvenes, dichos valores se promueven con todos los participantes a través de la formación que se realiza en la casa y a través del trabajo con la comunidad. A pesar de ser un movimiento mundial, se da autonomía a cada ciudad para trabajar en las necesidades particulares que se presenten en cada contexto. Para detectar estas necesidades en la Comuna 13 son los y las jóvenes voluntarios quienes a través de la manifestación de los problemas y de diferentes talleres de reconocimiento del territorio identifican las necesidades en sus barrios y dan las pautas a seguir para establecer qué problemas necesitan intervención. En la Comuna 13 el impacto que se quiere dar se centra en los y las jóvenes -siguiendo también la filosofía del movimiento- sin embargo, se trabaja en ocasiones con las familias de estos jóvenes voluntarios, sobre todo para el fortalecimiento del proyecto de vida. Asimismo, se trabaja con adultos, niños, población vulnerable, población desplazada, víctimas de Estado, víctimas de la violencia, entre otros, el trabajo se centra en lo comunitario y lo social.

El papel central de los y las jóvenes nace porque la persona que creó el movimiento Young Men's Christian Association, George Williams, en 1844 era un joven de 21 años y las primeras personas que hicieron parte de este también eran jóvenes, por esta razón el objetivo principal siempre ha sido incidir en esta población. (ACJYMCA, s.f.) Sin embargo, en la 'Y' Medellín, el papel principal de los jóvenes también se da porque en este territorio son los jóvenes quienes han demostrado que a través de diversas acciones pueden empezar a transformar su realidad y la de sus comunidades. Los y las jóvenes de la ' $Y$ ' Medellín buscan construir un mejor país, una mejor sociedad y saben que tienen el potencial y la capacidad de aprender de otros y están dispuestos a enfrentar retos y oportunidades.

A la Comuna 13, la 'Y' llega en los años 90, y en la casa actual, ubicada a pocas cuadras de la estación del metro San Javier, llevan alrededor de 30 años. Desde el primer momento de su presencia en el territorio, la ' $Y$ ' enfrentó todo el tema de la violencia que se daba en la Comuna, desde violaciones a derechos humanos hasta todo el 
tema de desaparecidos y desplazados. Fue entonces, como a partir de este reconocimiento de las problemáticas del territorio, se empezaron a indagar en las necesidades que se tenían en la Comuna y sobre todo en la población joven pues se necesitaba que ellos buscaran caminos diferentes a los que tenían a su alcance: la guerra, las armas y los negocios de narcotráfico. Es así como la casa de la ' $\mathrm{Y}$ ' empieza a convertirse no solo en un refugio físico para los y las jóvenes, sino en un lugar en donde además de sentirse seguros empiezan a formarse bajo el reconocimiento de sus propias habilidades y la formación de proyectos de vida alejados de la violencia.

El proceso de formación inicia entonces desde el interés de los y las jóvenes por participar, luego de ingresar se trabaja en el tema de habilidades para que cada uno se dé cuenta que sabe hacer diferentes cosas, que tiene muchas capacidades y que puede aportar a su comunidad. En la ' $Y$ ' es central el tema del ser y de la comprensión individual de habilidades y aptitudes para posteriormente poder transmitir esto a otras personas, allí es donde se incluye el tema de trabajo en equipo, comunicación asertiva y todo el tema de paz pues en la ' $Y$ ' existe gran interés porque los y las voluntarias sean gestores de paz. Dentro de la formación se abordan temas de equidad de género, diversidad sexual, derechos humanos, habilidades e inteligencias múltiples, recreación, se trabaja también el tema de la oralidad y del trabajo con otros reconociéndolos en su diferencia.

Actualmente, en base, la 'Y' Medellín tiene 50 voluntarios/as que se dividen en 2 grupos. Voluntarios/as jóvenes de 14 a 20 años y voluntarios/as universitarios de 21 a 28 años. Es importante mencionar que el proceso de formación se lleva a cabo a través de metodologías experienciales y metodologías de educación popular. Las actividades que se hacen para la enseñanza son actividades dinámicas y continuas lo que también permite que se empiece a generar un vínculo entre cada uno de los y las voluntarias de la ' $Y$ '. Como también lo pudieron contar Valeria, Gypsyn y Laura, integrantes del voluntariado de la ' $Y$ ', para quienes: 
Nuestro proceso de formación siempre ha sido desde lo vivencial y lo dinámico, principalmente nos han enseñado a que debemos de conocer la filosofía de la ' $Y$ ' $y$ de conocer todo ese ámbito social para después ir a generar acciones de incidencia y nos han formado para ser líderes, nos dan como bases para estructurar el cómo generar proyectos y cómo generar esos grupos tanto en la ' $Y$ ' como en diferentes organizaciones aliadas. (Entrevista Personal, Valeria Gutiérrez, 16 años. Voluntaria ACJ.)

La formación en la ACJ potencia las capacidades de cada persona independientemente de lo que a vos te guste hacer, lo que vos querás hacer potencia tus capacidades, si a ti te gusta hacer esto, está bien, hazle, iexplota! pero produce con esto que te gusta (...) si eres bueno en algo con seguridad vas a ayudar a otras personas. (Entrevista Personal, Gypsyn Quiroz, 16 años. Voluntaria ACJ.)

Yo creo que desde el voluntariado ya estamos generando cambios en la Comuna 13, porque a eso nos hemos dedicado: a desterrar malas hierbas conocidas como miedo, sembrando grandes flores conocidas como esperanza, siendo luz para todas esas personas que ya están perdiendo la suya y eso hace que la Comuna sienta un color diferente al blanco y negro de las balas y las víctimas. (Entrevista Personal, Laura Gil. Voluntaria ACJ.)

Dentro del voluntariado se tienen distintos rangos, estos son: verde, blanco, azul y rojo. Cada uno de los rangos comprende una serie de responsabilidades, estas se miden a través de un plan de adelanto que tiene la ' $\mathrm{Y}$ ' que mide las acciones que realizan los y las voluntarias. El primer rango es el verde, para obtenerlo es necesario reconocer y aceptar las bases y principios del movimiento, en lo posible apoyar el desarrollo de algún programa o proceso y participar activamente y con frecuencia en las actividades de formación que promueve la 'Y'. Para obtener el triángulo blanco, los y las voluntarias deben liderar un grupo, coordinar algún evento, 
tener conocimiento completo de la estructura del movimiento a nivel internacional, asistir a alguna sesión de la junta directiva y representar el movimiento en espacios de la ciudad. El triángulo azul se obtiene cuando se es parte del proceso de formación de otro compañero/ra que se encuentre en el rango de triángulo verde, a su vez, se debe coordinar campañas de recaudación de fondos, crear actividades donde se dé a conocer la misión y la filosofía del movimiento, acompañar y orientar grupos, asistir a las asambleas y seguir representando ' $\mathrm{Y}$ ' en espacios de la ciudad. Por último, para el triángulo rojo es necesario vincular $y$ seguir en el acompañamiento de personas nuevas, desarrollar actividades especiales sobre liderazgo o gobernanza, participar y difundir los programas y estar en espacios de influencia con otras instituciones.

Es importante mencionar que en la ' $\mathrm{Y}$ ' existen dos figuras: el voluntario y el colaborador. El colaborador, son todos los y las jóvenes que están en formación pero que todavía no tienen su triángulo verde, es decir el primero. Pero el colaborador también son todas las personas que hacen una actividad en la ' $\mathrm{Y}$ ' de manera voluntaria pero que no están en el proceso de formación. Para ser voluntario, es necesario que cada joven se identifique con la filosofía del movimiento, conviva y se relacione en un ambiente de fraternidad, así como que sea capaz de tomar decisiones, solucionar problemas y afrontar retos. En la ' $Y$ ' los voluntarios deben contar con salud mental y espiritual, deben saber reconocer sus habilidades y capacidades, así como ser conscientes del papel que cada uno de ellos/as cumple en su comunidad. Sus actitudes y comportamientos deben ser ejemplares porque ejercen desde su posición de voluntarios/as roles educativos, por lo que muchas veces son tomados como modelos a seguir.

Decidí quedarme porque me sentía en un espacio tranquilo, agradable, en donde me podía conectar, compartir con otra persona y en donde sentía empatía y amor por mi comunidad, por mi ciudad y mi país y todo porque las habilidades que a uno el movimiento ' $Y$ ' le entrega son habilidades que no solo le sirven para uno ejecutar en la vida de uno sino para uno 
empezar a ejecutarlas a nivel de ciudad, a nivel de Comuna, a nivel de país (...) las actividades pienso que son fundamentales para uno querer transformar y pues eso era lo que a mí me interesaba la transformación. (Entrevista Personal, Valeria Gutiérrez, 15 años. Voluntaria ACJ.)

Según Giraldo \& Ruiz (2019) en los jóvenes de las Comunas de Medellín, existe un vínculo de solidaridad especial y los vínculos sociales se fortalecen a través del compartir experiencias y saberes diversos, así como el valorar la singularidad de cada uno, las capacidades y potencialidades que tengan y desde las cuales pueden aportar a la construcción del proyecto común que se tenga. Con el acercamiento a campo se pudo evidenciar que dentro de la casa de la 'Y' existe un reconocimiento del otro como compañero y como par, asimismo se notó que todos pueden compartir sus conocimientos y experiencias y dentro del grupo son escuchados. De esta forma podría decirse entonces que la relación de los voluntarios también esta mediada por la reciprocidad, definida como: "la regla que crea consenso, porque descansa en un principio de equilibrio en las relaciones sociales" (Rosanvallon, 2015, 331). Al tener presente que todos están reunidos bajo el mismo objetivo, transformar su Comuna y sus barrios, el vínculo social que tienen se hace más fuerte y cobra mayor sentido en la medida que todos quieren lograr lo mismo. Lo anterior, implica identificarse con el otro como individuo y como parte de una comunidad.

Esta identificación y reconocimiento del otro es una particularidad que se tiene dentro de los procesos de voluntariado que según Giraldo \& Ruiz (2019) es la forma de solidaridad más extendida en el mundo. A partir de las diferentes acciones colectivas que se realizan dentro del voluntariado lo que se obtiene es una satisfacción y gratificación personal. Se confirma que entre las principales motivaciones de los voluntarios se encuentran: el interés por la comunidad, la transformación social, búsqueda de la reciprocidad y valores religiosos (Chacón et al., 2010, 220). Así como el fortalecimiento de la autoestima y la mejora del estado de ánimo. Para Manuela, coordinadora del voluntariado todas estas 
habilidades se fortalecen dentro de la ' $\mathrm{Y}$ ' y de esta forma se convierten en gestores de paz dentro de la Comuna 13:

Nosotros nos centramos en el tema del ser y en el tema de que los chicos, comprendan cuáles son sus habilidades, sus aptitudes y tengan todas las capacidades para difundir, para llevarle esas habilidades a otras personas, entonces involucramos todo el tema de trabajo en equipo, comunicación asertiva, y todo el tema de paz, nos interesa mucho que los chicos sean gestores de paz. (Entrevista Personal, Manuela Henao, 24 años. Coordinadora Voluntariado ACJ Medellín.)

\subsection{Ser joven de la ' $Y$ ': acciones colectivas}

La ' $\mathrm{Y}$ ' y sus voluntarios y voluntarias realizan diferentes acciones colectivas encaminadas al cumplimiento de diversos objetivos. Allí, las acciones colectivas están condicionadas, recreadas y dinamizadas por elementos como el contexto de exclusión socioeconómica y de conflictividad violenta que las enmarca (Alzate, 2012) esto, como se mencionó anteriormente por la historia y la memoria que tiene el espacio de la Comuna 13, así como las problemáticas actuales que se viven y han sido mencionadas. A su vez, dichas acciones colectivas, de acuerdo con Melucci (1990), construyen sistemas emergentes de cultura política que se entretejen con la vida diaria, proveen nuevas expresiones de identidad y van en oposición directa al orden dominante. Las acciones colectivas son entendidas entonces como las define Melucci (1999) es decir como una conjunción de individuos que se unen para actuar de manera colegiada por una motivación compartida o persiguiendo un fin.

En la 'Y' se puede afirmar que como menciona Park (1999) existe un sentimiento local, pues de la residencia parte la base de la participación en asuntos públicos y esta vecindad -la ' $Y$ '- se puede entender como una unidad social y como un espíritu colectivo. Asimismo, es importante "comprender la construcción social de la 
ciudad, de lo urbano y sus lugares, a través de los sujetos que la habitan corporal y emocionalmente" (Lindón, 2009, 6).

Chihu (2008) menciona que la emergencia de la acción colectiva no se explica únicamente por tensiones estructurales o conflictos ideológicos pues también depende de los recursos materiales, es decir, trabajo, dinero, beneficios concretos, servicios y de recursos no materiales como la autoridad, el compromiso moral, fe, amistad y cercanía que estén dentro de un determinado grupo. A su vez, el autor afirma que los actores que llevan a cabo una acción colectiva generalmente se identifican en términos del interés por un territorio, por la construcción de espacios para la actividad social y por la mejora de condiciones de vida. Esto es lo que ocurre precisamente en la ' $Y$ ', pues quiénes pertenecen a la casa tienen unos intereses en común que se resumen en las acciones colectivas que se llevan a cabo conjuntamente dentro de los diferentes barrios de la Comuna 13, además, a través del proceso de formación se genera un vínculo con todos los voluntarios y voluntarias como se explicó anteriormente.

Todas estas acciones cobran un mayor sentido por generarse dentro de un espacio urbano, sin embargo, es importante mencionar que según Lindón (2009), existen lugares en los cuales algunos sujetos ejercen o han ejercido la violencia en otros tiempos -caso de la Comuna 13-constituyendo una memoria del lugar.

En efecto, la Comuna 13 presenta una memoria de violencia, sin embargo, desde hace varios años lo que han intentado la mayoría de los habitantes de la Comuna 13 y en este caso particular los y las voluntarias de la ' $\mathrm{Y}$ ' es transformar esas memorias de lugar de violencia por un lugar que sea reconocido como resiliente y con gran potencial. Es entonces como "el espacio urbano representa para el hombre la tentativa más coherente y, en general, la más satisfactoria de recrear el mundo en que vive de acuerdo con su propio deseo (...) al crear la ciudad el hombre se recrea a sí mismo" (Park, 1999, 115). Además, es importante mencionar que el espacio es político y 
abierto a una lucha dentro de él, el espacio no es fijo, muerto ni neutral (Delgado, 2003, 136).

Dentro de las ciencias sociales y en especial en la sociología, el concepto de espacio reviste un valor importante para la comprensión de la sociedad y los procesos que se dan en ella. Para la sociología, el valor del espacio se define en términos de su carácter estructurante, pues organiza nuestras percepciones y las que tenemos de los fenómenos sociales, a la vez que es portador de acontecimientos sociales y de expresiones sobre la sociedad (Leal, 1997). Autores como Giddens (1984) plantean la necesidad de desarrollar la noción de lugar como la manifestación espacial de un hecho social. Los planteamientos anteriores son muestra de cómo, entre espacio y sociedad no solo existe un vínculo irrompible, sino una relación de orden recursivo de mutua influencia (Kuri, 2013) lo que también se hace notorio en la Comuna 13 y en la ' $Y$ '.

Asimismo, se necesita tener presente que la ciudad y la vida urbana son un proceso constante y complejo de construcción socioespacial de los lugares por parte de los sujetos (Lussault, 2007). Y es necesario reconocer que "la sociedad es producida y/o reproducida constantemente por los sujetos. En este devenir, el concepto de sujeto social reconoce un vínculo directo con la acción (Lindón, $2009,7)$. Además, es el sujeto social -los jóvenes voluntarios y voluntarias de la ' $\mathrm{Y}$ '- quien cuenta con la capacidad para transformar su realidad, crear nuevas estructuras, incluso a partir de las previas y transgredir lo antes aceptado, para innovar (Lindón, 2009).

Puede entonces afirmarse que la ' $Y$ ' como menciona Vidal (2015) apela a una idea de territorialidad definida $-y$ de identidad con ese territorio-, lo anterior para generar una defensa o transformación espacial en donde le otorgan sentidos y significados particulares al territorio a través de su deseo de transformación y los objetivos que acompañen al mismo.

Pero ¿por qué la población joven? Según Urán (2002) la juventud se define como una categoría social y cultural que denota un grupo 
social etario, pero que se caracteriza por la apertura a la experimentación y la curiosidad de lo nuevo. A su vez, es definida como un grupo que cuestiona la realidad y como un agente de cambio y transformación social, política y cultural. Para él, la espacialidad es un asunto de primer orden que define la construcción de la identidad social del joven, pues cada grupo o cultura juvenil se configura dentro de un entorno territorial y gran parte de su identidad se construye por medio de la interacción que este tiene con los diferentes referentes materiales y simbólicos que lo rodean constantemente.

\subsection{Las experiencias en la ' $Y$ ': PAZA LA PAZ}

PAZA LA PAZ es uno de los programas con los que cuenta la ' $Y$ ', su objetivo es generar oportunidades para los y las jóvenes, que están en situación de vulnerabilidad social, para propiciar la inclusión, empoderamiento e incidencia en la construcción de paz de Colombia; esto a través de un modelo de educación popular constructivista y con propósito de proyección social. En Medellín el programa está hace 4 años aproximadamente y ha impactado la vida de cerca de 400 jóvenes de manera positiva en estos temas, sin embargo, entre sus metas está el impactar positivamente la vida de más de 1530 jóvenes (personas entre los 14 y 28 años) 55\% mujeres, $45 \%$ hombres, $87 \%$ en zonas urbanas y $13 \%$ en la ruralidad de todo el departamento de Antioquia. Los temas principales que trata el programa son: convivencia y paz, emprendimiento, organización y participación juvenil, equidad de género, inclusión, empoderamiento de la mujer, etc.

El proyecto está compuesto por cuatro ejes de formación y cada eje cuenta con objetivos, indicadores y resultados. Los cuatro ejes son: convivencia y paz, organización y participación juvenil, emprendimiento $\mathrm{y}$ fortalecimiento institucional. El primer componente, de convivencia y paz tiene por objetivos que los y las jóvenes ofrezcan soluciones pacíficas como gestores de paz y convivencia en sus familias y contextos. Asimismo, se busca que los y las jóvenes se conviertan en líderes gestores de paz y convivencia 
en sus familias y territorios. Como indicador, se busca que a diciembre 30 de 2020 al menos el $80 \%$ de los y las jóvenes formados en equidad de género y convivencia y paz con análisis pre y post en la escala de liderazgo, incrementan su autoestima, habilidades sociales y toma de decisiones asertivas que ayuden a la construcción de su proyecto de vida. El resultado: se espera que los y las jóvenes fortalezcan sus habilidades sociales y su sentido de vida. Asimismo, afirma Andrés, director del programa que: El proceso de formación que se expande más a articular a otros lugares de la ciudad y eso que aprenden van y lo replican con otros jóvenes para que ese grupo de líderes genere reflexiones en otros grupos y ese grupo fortalezca conocimientos y luego vaya y replique. (Andrés Vargas. Entrevista Personal. Promotor de participación y organización juvenil.)

El segundo componente, organización y participación juvenil tiene como objetivos que los y las jóvenes fortalezcan su ciudadanía y participan e inciden en espacios locales, departamentales y/o nacionales en juventud, así como que los y las jóvenes formados en mecanismos de participación y en espacios políticos de incidencia ejerzan su ciudadanía. Como indicador, a diciembre de 2020, por lo menos el $20 \%$ de los y las jóvenes se vinculen a diferentes espacios de organización, representación y agendas públicas en sus territorios en temas de jóvenes, paz y equidad de género. Y como resultado, los y las jóvenes son formados en mecanismos de participación y en espacios políticos de incidencia.

El tercer componente, emprendimiento, tiene como objetivos que los y las jóvenes participan en emprendimientos sociales y económicos que permiten fortalecer sus habilidades sociales y su sentido de vida. Los y las jóvenes formados en planeación y gestión, educación financiera, pensamiento creativo, planes de negocio, formulan propuestas de emprendimiento social o económico. Como indicador es que a diciembre de 2020 los y las jóvenes desarrollan 21 iniciativas de emprendimiento social o económico por año y que por lo menos de 21 propuestas formuladas por los jóvenes reciban capital semilla para su implementación y aportan a los proyectos de vida de los y las jóvenes. 
El cuarto y último componente referido a fortalecimiento institucional, tiene como objetivos cualificar prácticas sociales de la ACJ Medellín, para el trabajo con paz, género, juventud y emprendimiento. Asimismo, se conformó un comité para gestionar recursos en donde se consoliden propuestas y proyectos con diferentes organizaciones para trabajar conjuntamente en estos temas. Entre los indicadores, se buscó que al finalizar se tuviera un paquete de productos y servicios que se relacionen con la promoción de paz, así como poder tejer redes y encontrar aliados para aportar a la construcción de paz en el país.

La formación de los y las jóvenes que hicieron parte del proyecto se fundamentó en la hipótesis de cambio, que afirmaba que a partir del aprovechamiento de las herramientas de formación se consolidan proyectos de vida y las posibilidades para lograrlos, dejando de lado las actividades ilegales y violentas. Asimismo, a partir de la participación en el proyecto se contribuye a la construcción de paz y la transformación del conflicto social en Colombia, generando así oportunidades para el empoderamiento y la inclusión de la juventud. Los temas de cada sesión se dividieron en diversas competencias como: liderazgo juvenil, liderazgo transformacional, ser de relaciones grupales, trabajo en equipo, asertividad y resiliencia, comunicación, pensamiento creativo, cultura de paz, conflicto, entre otros.

Por otro lado, se puede afirmar que el programa de PAZA LA PAZ se articula directa e indirectamente con todos los otros programas de la ' $Y$ ', pues en el proceso de formación se busca enseñar que la paz también está en actitudes como el respeto al entorno, la recuperación de espacios, prevenir la destrucción de la naturaleza, el no contaminar y todo reducido a la responsabilidad social. Es aquí donde se vincula la actividad del Challenge, que se realizó el año pasado en uno de los barrios de la Comuna 13. Este reto se hace mundialmente en el cumpleaños de la ' $\mathrm{Y}$ ', el año pasado se cumplieron 275 años, por lo tanto, fueron 175 minutos de voluntariado en donde lo que se realizó fue una limpieza y descontaminación de basuras. 
Como se puede observar, el programa busca fortalecer cuestiones personales y del individuo. Dentro del programa se tiene una escala de liderazgo: una línea base pre y pos, en la que se evalúa el avance de los participantes del programa. Si bien muchos de los ítems que se evalúan se enmarcan en lo mencionado anteriormente, como menciona Andrés, director del programa PAZA LA PAZ de la 'Y': Uno a veces se queda mucho en lo personal, el crecimiento personal, el fortalecimiento de la persona y no sé qué y eso... pero también esos temas de lo político, de lo participativo de la incidencia, de la conceptualización de la norma es muy importante y también se ha convertido en un reto, es un reto. (Andrés Vargas. Entrevista Personal. Promotor de participación y organización juvenil.)

Entre otros de los talleres que se realizaron con los jóvenes de la ' $\mathrm{Y}$ ' sobre su papel como voluntarios, estos identificaron y definieron por medio de una lluvia de ideas lo que para ellos era construcción de paz, entre las palabras que más se destacaron fueron: compromiso, unión, proceso, amor, cooperación, comprensión, coordinación, claridad, reconciliación, convivencia, empatía, resiliencia, solidaridad, tranquilidad, paciencia, respeto, comunicación, bienestar, tolerancia, protección. Asimismo, identificaron que a partir de su rol como voluntarios pueden hacer que la comunidad se empodere y empiece a realizar acciones colectivas para transformar las realidades que quieren transformar. Lo anterior a partir del sentido de pertenencia que pueden generar, así como el entusiasmo, la voluntad, buena comunicación y la concientización que generan con sus acciones.

\section{Conclusiones}

Como se pudo evidenciar durante el trabajo de campo y con el acompañamiento a las actividades llevadas a cabo por la ' $Y$ ' durante el año 2019, se pudo identificar que efectivamente, sí se fortalecen las capacidades como individuos de cada uno de los voluntarios, además se estimula el liderazgo a través de metodologías experienciales y metodologías de educación popular. Asimismo, se pudo reconocer el papel importante que tiene la ACJ dentro de la 
Comuna 13, no solo por su labor con los y las voluntarias sino también por el reconocimiento y las actividades de impacto e incidencia que son vistas por los demás habitantes de la Comuna 13.

Por otro lado, se dejó en evidencia la importancia que tienen los y las jóvenes dentro del ejercicio de transformación de la Comuna 13, pues en el recorrido previo que se hizo por la Comuna y el reconocimiento de esta, se pudo notar que existen diferentes iniciativas que buscan generar cambios. Estas agrupaciones, están en su mayoría, compuestas por jóvenes. Se pudo tener el acercamiento a organizaciones como: Zippy Tour, que nace de la unión de 4 jóvenes que viven en la Comuna 13 y realizar tours guiados por las escaleras eléctricas y los grafitis de la Comuna; Black and White $\mathrm{C} 13$, un grupo artístico de jóvenes de una de las 4 ramas del Hip-Hop ubicados en el tramo 6 de las escaleras eléctricas; Casa Kolacho, un centro cultural de HipHop que promueve la enseñanza de diversos tipos de arte; SonBatá, colectivo artístico de la Comuna enfocado en la música, el baile y la cultura, entre otros.

Todo lo que estas iniciativas tienen en común es el deseo por poder mostrar una cara no violenta de la Comuna 13, así como generar espacios dentro del territorio para el reconocimiento de diversos tipos de arte. Muchos de los y las voluntarias reconocen que el hecho de vivir en un lugar que ha estado teñido con memorias y altos índices de violencia hace que cada vez, tengan más deseos por incidir en su Comuna a través de diferentes intervenciones de una forma positiva, así como afirman que piensan que si no hubieran nacido en un lugar con estos problemas no habrían tenido tanto interés en temas sociales.

La ' $Y$ ' al verse como una unidad social y como un grupo que tiene un espíritu colectivo busca transformar su realidad y la de la Comuna 13 a través no solo de la educación, la réplica de conocimiento y el liderazgo sino también a partir de las acciones colectivas que se realizan en el marco del deseo de vivir en un territorio que resalte la libertad, dignidad, el amor y diversos valores. Este grupo de jóvenes voluntarios y voluntarias ven el pertenecer a una organización como 
la ' $Y$ ' como: "un acto de rebeldía frente a aquello que impide seguir existiendo (Zemmelman \& Quintar, 2005, 45). Lo anterior, permite confirmar la afirmación: al crear la ciudad el hombre se recrea a sí mismo" (Park, 1999, 115).

En cuanto a la organización y administración de la ' $\mathrm{Y}$ ' también existen múltiples ventajas y es que los y las voluntarias pueden estar al tanto de las decisiones que se toman dentro de la casa, aparte de ser uno de los requerimientos para poder subir de rango en el voluntariado también se busca dentro de la organización que todas las voces puedan influir y manifestar sus observaciones para así poder mejorar constantemente. Muestra de lo anterior, es la experiencia que ha tenido con la ' $\mathrm{Y}$ ' la actual coordinadora del voluntariado Manuela Henao, quién lleva 6 años en la organización de los cuales 2 fue voluntaria, 3 hizo parte del staff, también realizó su práctica profesional de trabajo social en la organización y actualmente es líder de los programas de voluntariado, lo que muestra la oportunidad de crecer dentro de la organización.

Al ser los y las jóvenes quiénes identifiquen las necesidades que tienen, son ellos mismos quienes deciden cuales son los aspectos que quieren intervenir y cambiar de sus propios barrios. La importancia que se le da a los y las jóvenes dentro de la organización hace que ellos/as mismas se empoderen y sean quienes vean el potencial y todos los cambios que pueden realizar en el presente bajo el objetivo de construir el futuro que quieren. Asimismo, se les da la certeza que son ellos/as mismas quienes tienen que trabajar para la construcción de un mejor país, empezando desde su comunidad más cercana, es decir, su barrio -la Comuna 13-. Dentro de la ' $Y$ ' lo que se rescata constantemente es el potencial de los y las jóvenes y esa capacidad que tienen de aprender de otros, de no juzgar, de estar dispuestos a enfrentar nuevas oportunidades, recibir opiniones y ejercer su papel como ciudadanos activos.

Por otro lado, la enseñanza que se brinda en temas de: equidad de género, diversidad sexual, derechos humanos, habilidades, inteligencias múltiples, oralidad, trabajo en equipo, recreación y 
potencialización de diferentes habilidades, permite que los y las voluntarias se formen bajo una enseñanza integral en la que puedan aprender a desarrollar valores como la empatía, tolerancia, participación, respeto, unidad, entre otros, que lo que hacen es completar la formación de líderes que la ' $Y$ ' desea obtener. Esta educación y la habilidad que con ella adquieren no solo sirven para ser ejecutadas en la vida de cada uno sino también para empezar a aplicarlas a nivel ciudad, nivel Comuna y nivel país. Lo anterior, a través de la construcción de puentes que generen empatía, servicio y comunicación con otras personas, así como desde las acciones más pequeñas o simples que involucren a la comunidad en espacios culturales y educativos, o en la generación de ámbitos artísticos.

El ser voluntario/a de la ' $\mathrm{Y}$ ' ha hecho que muchos jóvenes se interesen por aspectos como el tejido social, la construcción conjunta de saberes y el amor por la comunidad. Esto porque el espacio en la casa de la ' $Y$ ' hace que muchos de ellos se empiecen a involucrar en espacios y realidades totalmente diferentes a las que habitan en su cotidianidad. Con lo anterior, se empiezan a dar cuenta que dichos espacios de participación les permiten empezar a reconocer al otro como igual, partiendo de la idea de que cada uno de ellos existe en un entorno que no solo tiene las mismas problemáticas sino también el mismo potencial de transformación a partir de la unión de ideas y esfuerzos -acciones colectivas-. Como se mencionó en uno de los talleres de aprendizaje: "los cambios no se hacen por arte de magia, sino por arte de barrio"

\section{Bibliografía}

Asociación Cristiana de Jóvenes. Medellín. (2017). ACJ . YMCA Medellín.https://issuu.com/acjymcamedellincol/docs/informe social 2017 49ba1ad57d $\underline{40 \mathrm{~b} 8}$

Asociación Cristiana de Jóvenes. Medellín. (2016). PAZA LA PAZ: Líderes Juveniles que Transforman y Emprenden Malla conceptual para el módulo de formación. Horyzon. 
Álzate, M. (2012). Acciones colectivas frente a la violencia. Disquisiciones a partir de un estudio de casos: Comuna 13 de Medellín (Colombia). Opinião pública, Campinas, vol. 18, $2,427-451$

Asociación Cristiana de Jóvenes. (s.f.). Conócenos: HISTORIA YMCA. http://ymcamedellin.org/conocenos/

Asociación Cristiana de Jóvenes. (s.f.). Conócenos: EL MOVIMIENTO YMCA. http://ymcamedellin.org/conocenos/

Asociación Cristiana de Jóvenes. (s.f.). Conócenos: ACJ-YMCA MEDELLÍN. ¿Quiénes somos? http://ymcamedellin.org/conocenos/

Centro Nacional de Memoria Histórica. (2011). La huella Invisible de la guerra: desplazamiento forzado en la comuna 13. Taurus.

Chacón, F., Pérez, T., Flores, J., y Vecina, M. (2010). Motivos del voluntariado: categorización de las motivaciones mediante pregunta abierta. Intervención Psicosocial, 19(3), 213-222.

Chihu, A. (2008). Melucci: la teoría de la acción colectiva. UNAM.

Cinep, Justicia \& Paz. (2003) Noche y Niebla y violencia política en Colombia. Caso tipo $N^{\circ} 2$. Banco de datos de violencia política. Programa Andino Democracia y Derechos Humanos.

Comisión Nacional de Reparación y Reconciliación. Grupo de Memoria Histórica (2013) iBASTA YA! Colombia: Memorias de guerra y dignidad. Imprenta Nacional. Capítulo II

Consejo Mundial de ACJs. (1998) Desafío XXI. 14 Consejo Mundial de ACJ's.

Consejo Mundial de Kampala. (1973). Declaración de Kampala.

Delgado, M. (2003). Debates sobre el espacio en la geografía contemporánea. Universidad Nacional de Colombia. Red de estudios de Espacio y Territorio.

El Espectador. (11 Julio 2019) La Comuna 13 de Medellín exhibe su renacer ante autoridades locales del mundo.

https://www.elespectador.com/noticias/nacional/antioqu ia/la-comuna-13-de-medellin-exhibe-su-renacer-anteautoridades-locales-del-mundo-articulo-870523 
El Tiempo. (11 Julio 2016) Graffitour, un recorrido por la transformación de la comuna 13. Por: David Calle Atehortúa.

https://www.eltiempo.com/archivo/documento/CMS$\underline{16641686}$

Giddens, A. (1984). La constitución de la sociedad. Bases para la teoría de la estructuración. Amorrortu Editores

Giraldo, Y. \& Ruiz, A. (2019). La solidaridad. Otra forma de ser joven en las comunas de Medellín. Universidad Pedagógica Nacional; Consejo Latinoamericano de Ciencias Sociales, Universidad de Manizales; Fundación Centro Internacional de Educación y Desarrollo Humano.

Gutiérrez, F. (2015) "¿Una historia Simple?" En:Comisión Histórica del Conflicto Armado en Colombia. Contribución al entendimiento del conflicto armado en Colombia. La Habana: Mesa de Negociación entre el Gobierno Nacional y las Fuerzas Armadas Revolucionarias (FARC). 498-540

Kuri, E. (2013). Representaciones y significados en la relación espacio-sociedad: una reflexión teórica. Sociológica (México), 28(78), 69-98.

http://www.scielo.org.mx/scielo.php?script=sci arttext\&p $\mathrm{id}=$ S0187-01732013000100003\&lng=es\&tlng=es

Leal, J. (1997). Sociología del espacio: el orden espacial de las relaciones sociales. Política Y Sociedad, 25, 21. https://revistas.ucm.es/index.php/POSO/article/view/PO $\underline{\text { S09797230021A }}$

Lindón, A. (2009). La construcción socioespacial de la ciudad: el sujeto cuerpo y el sujeto sentimiento. Revista Latinoamericana de Estudios sobre Cuerpos, Emociones y Sociedad, 1(1),6-20. ISSN: 1852-8759. https://www.redalyc.org/articulo.oa?id=273220612009

Lussault, M. (2007). L'homme spatial: la construction sociale de l'espace humain. Seuil.

Martin, G. (2014) Medellín, tragedia y resurrección. Mafias, ciudad y Estado. 1975-2013. Editorial La Carreta. 
Martin, G. (2019). Memorias y violencias en Medellín. HiSTOReLo. Revista de Historia Regional y Local, 11(22), 340-368. https://doi.org/10.15446/historelo.v11n22.78131

Melucci, A. (1990). La acción colectiva como construcción social. Ponencia. Clases Sociales y Movimientos Sociales. Congreso Mundial de Sociología, Madrid.

Melucci, A. (1999). Acción colectiva, vida cotidiana y democracia. México, D.F.: El Colegio de Mexico. http://www.doi.org/10.2307/i.ctvhn0c2h

Moreno, R. (2003). Conflicto y violencia urbana en Medellín desde la década del 90: algunas valoraciones. Instituto Popular de Capacitación.

Park, R. (1999) La ciudad como laboratorio social. En R. Park La ciudad y otros ensayos de ecología urbana. (pp. 115-126). Ediciones del Serbal.

Rosanvallon, P. (2015). La sociedad de iguales. Manantial.

Semana. (2013) Comuna 13: "En este lugar florecen el turismo, la autonomía y la integración social". Revista Semana. https://www.semana.com/cultura/multimedia/comuna13-en-este-lugar-florecen-el-turismo-la-autonomia-y-laintegracion-social/650881

Urán, O. (2009). Participación ciudadana y transformación democrática del conflicto urbano. Notas a propósito del Programa de Presupuesto Participativo en la Ciudad de Medellín, Colombia. Cuadernos IPPUR/UFRJ, 23(1), 175200.

Urán, O. (2002) Ciudadanía y Juventud. Constitución de los jóvenes en sujetos ciudadanos. JOVENes, Revista de Estudios sobre juventud. Edición Nueva Época, año 6, núm. 16.

Verdad Abierta (17 de Octubre 2018) General Montoya: diga la verdad sobre la Operación Orión": víctimas de Comuna 13. https://verdadabierta.com/general-montoya-diga-laverdad-la-operacion-orion-victimas-comuna-13/

Vidal, D. A. (2015). Pensar la formación para la ciudadanía en clave espacial. Una lectura comprensiva de David Harvey y 
Doreen Massey. Revista Colombiana de Sociología, 38(1), 165-182.

Zemmelman, H., y Quintar, E. (2005). Pedagogía de la dignidad de estar siendo. Entrevista con Hugo Zemmelman y Estela Quintar a cargo de Jorge Rivas. http://www.fisicanet.com.ar/carreras cursos/articulos ed ucativos/28 07 1.php

\section{(c) $(1)$}

Este texto está protegido por una licencia Reconocimiento Creative Commons 4.0.

Usted es libre para Compartir - copiar y redistribuir el material en cualquier medio o formato- y Adaptar el documento — remezclar, transformar y crear a partir del material - para cualquier propósito, incluso comercialmente, siempre que cumpla la condición de:

Atribución: Usted debe reconocer el crédito de una obra de manera adecuada, proporcionar un enlace a la licencia, e indicar si se han realizado cambios. Puede hacerlo en cualquier forma razonable, pero no de forma tal que sugiera que tiene el apoyo del licenciante o lo recibe por el uso que hace. 